\title{
Lateral squeezing effects on cement-slag-bentonite slurry wall performance
}

\author{
Davood Talefirouz ${ }^{1 * *}$, Erdal Çokça ${ }^{2}$ \\ ${ }^{1}$ Civil Engineering Department, Middle East Technical University, Ankara, Turkey \\ ${ }^{2}$ Civil Engineering Department, Middle East Technical University, Ankara, Turkey \\ *Corresponding author: E-mail: talefirouz@gmail.com
}

\begin{abstract}
English
Cement-slag-bentonite slurry walls are self-hardening structures, and they are mainly used to retard contamination transport into the groundwater stream. Whilst permeability of a mixture is an initial criterion in slurry wall design and material selection, long-term performance is mainly influenced by curing ages and stress-state caused by adjacent soil. In this study, the steady-state of effective stresses at 7 days and 28 days of curing age is predicted. The effect of the modulus of horizontal subgrade reaction, interface friction, and transition of the earth pressure from at-rest to the active condition was applied to develop the model. Unlike the quantities that the geostatic model presented, this method gives a slight decrease of stresses after a certain depth, and the trend is in good agreement with trends provided by previous studies. Furthermore, the predicted stresses are then applied to estimate the permeability of the wall at each depth and compare it with those obtained in the laboratory. Finally, predicted effective stresses stay lower than geostatic stress, and the slurry wall consolidation along with the sidewalls' lateral squeezing leads to keeping the stress state under control.
\end{abstract}

Keywords: cement-slag-bentonite, compressibility; lateral squeezing, permeability, slurry wall

\section{Introduction}

Cement-slag-bentonite (CSB) slurry walls as nonstructural barrier techniques have been widely employed to decrease contaminants' migration into the groundwater stream. Within this area of consideration, a proper estimation of the wall permeability is an important parameter for the slurry wall design. For groundwater control applications, a permeability of $1 \times 10^{-9}$ $\mathrm{m} / \mathrm{s}$ is specified for CSB slurry walls, although a permeability of $1 \times 10^{-8} \mathrm{~m} / \mathrm{s}$ or higher would be sufficient for most remediation applications [1]. Even though the self-hardening property of the CSB mixture exerts effects on permeability reduction, several studies have also shown that permeability decreases as the weight of the joint increases [2-4]. Consequently, an appropriate prediction of the lateral earth pressure development within the slurry wall is important. The hypothesis of the lateral squeezing modeling was initially proposed by Filz [5], and this method for modeling was then developed by many researchers considering the arching and squeezing effect for deep trenches [6-8].

Observations differ for the analysis of the mechanism; however, the results of all approaches indicate that the stress state in the trench (below the depth of 5-7 m) remains lower than the geostatic approach. It has been suggested that the major principal stress in deep-narrow slurry walls is horizontal, and it is vertical in shallow-wide slurry walls $[5,9$, 8]. However, the specific mechanism behind the lateral squeezing models $[9,5]$ has not been fully addressed for modeling of one sort or another. Proposed theories are based on the 
assumption that either the earth pressure coefficient is in the at-rest condition for surrounding soils or neglects the down-drag force between the mixture placements and completed consolidation.

This paper describes a model to estimate effective stress in CSB slurry walls employed at 7 days and 28 days of curing ages based on laboratory consolidation and index tests. To consider both arching and lateral squeezing mechanisms, the method provided by Li et al. [6] is used to obtain major principal stress (horizontal stress) with depth. Earth pressure transition from at-rest to the active condition presented by Bang and Kim [10] is then applied to evaluate minor principal stress (vertical stress). Then, the permeability of the wall can be predicted based on obtained stresses at a given curing age and compared with those achieved in the laboratory for the same stress values.

\section{Material and Methods}

To identify the stress-state in a CSB slurry wall, two different mixtures with $20 \%$ of cementitious material mixed with $80 \%$ of the bentonite-water slurry are used. For the first mixture, ground granulated blast furnace slag (GGBS) is replaced with 50\% cement type I by dry weight to make up the $20 \%$ of cementitious material. In this mixture, the bentonite-water slurry contains $5 \%$ of bentonite mixed into distilled water to make up the $80 \%$ batch slurry which is allowed to hydrate for one week. Hence, one kilogram of this mixture comprises the following: $100 \mathrm{~g}$ cement, $100 \mathrm{~g}$ GGBS, $40 \mathrm{~g}$ bentonite, and 760 $\mathrm{g}$ distilled water (C50-S50-B5). In the second mixture, $5 \%$ lime is employed for the cementitious mixture mentioned above and replaced with a $5 \%$ portion of GGBS as well as increasing the bentonite content of the batch slurry to $9 \%$. Consequently, one kilogram of this mixture includes the following proportions: $100 \mathrm{~g}$ cement, $90 \mathrm{~g}$ GGBS, $10 \mathrm{~g}$ lime, $72 \mathrm{~g}$ bentonite, and $728 \mathrm{~g}$ distilled water (C50-S45-L5-B9). The following chemical reactions are expected among the materials in the mixture. The lime in a hydrated form
$\left(\mathrm{Ca}(\mathrm{OH})_{2}\right)$ reacts with the montmorillonite structure containing alumino-silicates and also reacts with GGBS, which is a pozzolanic material and has $\mathrm{CaO}, \mathrm{SiO}_{2}$, and $\mathrm{Al}_{2} \mathrm{O}_{3}$. These silica and aluminum particles react with lime in a hydrated structure to form calcium silicate hydrate (CSH), calcium aluminate silicate hydrate $(\mathrm{CASH})$, and calcium aluminate hydrate $(\mathrm{CAH})$, which are separated into gap minerals in response to calcium particles. CSA and CAH are cementitious objects, such as those in the form of a Portland concrete grid $\left(3 \mathrm{CaO} \cdot \mathrm{SiO}, 2 \mathrm{CaO} \cdot \mathrm{SiO}_{2}, 3 \mathrm{CaO} \cdot \mathrm{Al}_{2} \mathrm{O}_{3}\right.$, $4 \mathrm{CaO} \cdot \mathrm{Al}_{2} \mathrm{O}_{3} \cdot \mathrm{Fe}_{2} \mathrm{O}_{3}$ ). The matrix formed is permanent. The state of stress in slurry walls depends on inward pressure from adjacent soils and the weight of the wall [5, 11]. A two-dimensional CSB slurry wall, with width and depth of $B$ and $H$, respectively, surrounded by submerged medium dense sand, is investigated in this paper. It is assumed that the mixture placed into the trench along with the surrounding soils is homogeneous and isotropic, and the groundwater table is at the surface [5-8]. Since the horizontal strain of mixtures after placement is expected to be zero, the geometry of the problem is identified as plain-strain. Subsequently, in this two-dimensional coordinate system, inward horizontal deformation is assumed to be positive, and the ground surface is indicated as the origin of $\mathrm{z}$ (Figure 1). Consequently, in the following problem, the longitudinal direction implies the transverse direction. Once the fresh mixture is placed, it is assumed that the pore water pressure $u=\gamma_{\mathrm{w}} z+\gamma_{\text {csb }}^{\prime} \mathrm{z}$ carries the self-weight of the CSB at depth $\mathrm{z}$ of the wall and at excess pore water pressure $u_{e}=\gamma_{\mathrm{csb}}^{\prime} \mathrm{z}$, where $\gamma_{\mathrm{w}}$ and $\gamma_{\text {csb }}^{\prime}$ are the unit weight of water and floating unit weight of CSB, respectively, and the vertical and horizontal effective stress in the wall is $\sigma^{\prime}=\sigma_{v}^{\prime}=0$. During the consolidation procedure, the pore water pressure is intended to decline to hydrostatic pressure, and the magnitude of effective stresses need to be specified.

According to Filz [5], inward displacement of the trench sidewalls should take place to keep horizontal stress equilibrium applied to the sidewalls. Alternatively, the interface frictional 


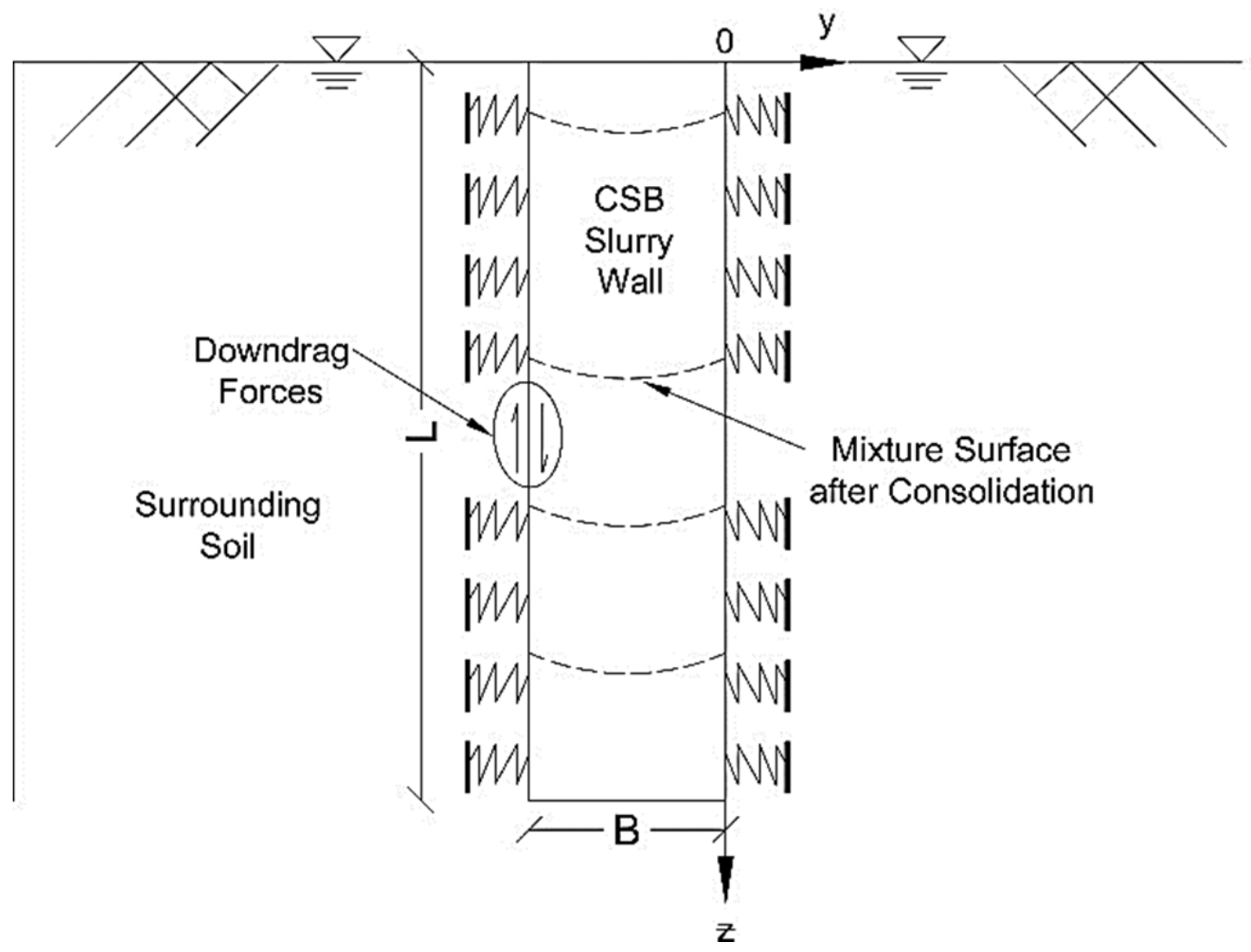

Figure 1: Schematic of the sidewall friction on the stress distribution in a CSB slurry wall.

stress $\tau$ between the trench wall and adjacent soil during the consolidation procedure $[3,6,7]$ follows the Mohr-Coulomb strength criterion as

$$
\tau=\mathrm{C}_{\text {in }}^{\prime}+\sigma_{\mathrm{h}}^{\prime} \tan {{ }^{\prime}}^{\prime}{ }_{\text {in }}
$$

where $C^{\prime}$ in and $\varnothing_{\text {in }}^{\prime}$ are the solidity and internal friction angle of the sidewall interface, respectively, and as mentioned by Potyondy [12], the relationship is as follows

$$
C^{\prime}{ }_{i n}=R C^{\prime}{ }_{C S B}
$$

and

$$
\tan \varnothing^{\prime}{ }_{i n}=\operatorname{Rtan} \varnothing^{\prime}{ }_{i n}
$$

where $C_{{ }_{C S B}}^{\prime} \emptyset^{\prime}{ }_{C S B}$, and $R$ are the cohesion, internal friction angle, and shear strength reduction factor of the cured wall, respectively. It is assumed that effective horizontal stress at the ground surface and the top of the CSB wall is $\sigma_{h}^{\prime}=0$ at $\mathrm{z}=0$. A model proposed by Li et al. [6] for a lateral squeezing effect caused by the inward displacement of side walls is

$$
\begin{aligned}
\sigma_{h}^{\prime}= & \frac{B{\gamma^{\prime}}^{\prime}{ }_{C S B}}{2 \tan \varnothing^{\prime}{ }_{i n}}\left(1+A-\frac{2{c^{\prime}}^{\prime}}{B{\gamma^{\prime}}^{\prime}{ }_{C S B}}\right) \\
& \times\left[1-\exp \left(-\frac{2 \tan \varnothing^{\prime}{ }_{i n}}{B D} Z\right)\right]
\end{aligned}
$$

where the coefficient of $A$ and $D$ can be outlined as

$$
A=\frac{2 E}{v(1+v) B K}
$$

so that

$$
D=\frac{1-v}{v}+A
$$

According to Das [13], an elastic medium of soils can be expressed as a series of infinitely 
elastic springs (Figure 1), and the subgrade module for cohesive soils at depth $\mathrm{z}$ can be characterized as

$$
k_{z}=n_{h} Z
$$

where $n_{h}$ is the constant of modulus of horizontal subgrade reaction. As reported by Filz [5], the following relationship between vertical and horizontal effective stress in the lateral squeezing model is

$$
\sigma^{\prime}{ }_{v}=k_{m} \sigma_{h}^{\prime}
$$

where $k_{m}$ is the coefficient of lateral earth pressure employed into the trench wall. The conventional consideration is that there is no need to evaluate wall-to-soil shear resistance or down-drag force when the coefficient of at-rest earth pressure is assumed for the surrounding soil [14]. However, during fresh mixture placement into the trench, the mixture will settle corresponding to the sidewall under its weight and deploys a down-drag force. This phenomenon then develops until consolidation completion. The role of an at-rest earth pressure condition in a slurry wall, that is, no inward displacement of surrounding soils into the trench, is assumed. Besides, Clough and Duncan [14] pointed out that where the proposed backfill material is cohesive, a moderate earth pressure between at-rest and active conditions must be mobilized for design. To illustrate lateral earth pressure transition from at-rest to active condition, a method proposed by Bang and Kim [10] is investigated to analyses the relationship between the major and minor principal effective stresses. Where $k_{0}$ is known as the coefficient of at-rest lateral earth pressure, the subsequent angle $\emptyset_{0}$ can be described as

$$
\sin \phi_{0}=\frac{1-k_{0}}{1+k_{0}}
$$

$$
\begin{aligned}
\text { where } k_{0} & =1-\sin \varnothing_{0} \text {, then } \\
\sin \phi_{0} & =\sin \phi /(2-\sin \phi)
\end{aligned}
$$

If the angle, $\psi$, refers to the relationship between major (horizontal) and minor (vertical) principal effective stress, then considering a $\psi$ value between $\varnothing$ and $\emptyset_{0}$ describes a displacement angle for active and at-rest values, respectively. So, the intermediate part at each depth can be defined as $\emptyset_{0}<\psi<\emptyset$. Furthermore, where $\delta$ is the cured slurry wall friction angle, so that;

$$
\sin \Delta=\sin \delta / \sin \varnothing
$$

Since $\psi(z)$ can be used instead of $\varnothing$, and $\delta$ $(\mathrm{z})$ is the developed cured slurry wall friction at depth z, Equation (11) can be modified to result in

$$
\sin \Delta(z)=[\sin \delta(z) / \sin \psi(z)]
$$

And the variation of $\delta(\mathrm{z})$ can be considered to be zero for at-rest and to be $\delta_{\text {max }}$ in an active condition. The average stress for plain-strain at any depth is

$$
\sigma=\frac{\sigma_{v}+\sigma_{h}}{2}
$$

Subsequently, the complete solution once $\sigma$ along the ground surface up to the entire depth (z), that is, developed at the face of the slurry wall, the intermediate earth pressure coefficient can be analyzed from

$$
k m=[\sin (\Delta(z)-\delta(z)) / \sin \Delta(z)] \cos \delta(z) \mid z=z_{y}
$$

However, Equation (14) requires detailed solution steps, which is a function of, $\psi(\mathrm{z})$, and provides lateral earth pressure transition from at-rest to the active condition. The variation for $\psi(\mathrm{z})$, is from $\emptyset_{0}$ for at-rest to $\emptyset$ for active condition. In other words, if the stage of the sidewall from mixture placement to consolidation completion denotes $\beta$, then $\beta=0$ and $\beta=1$ can describe the at-rest and active conditions, respectively (Figure 2). Accordingly, the deformation development of the surrounding soils at $\mathrm{z}=0$ is $0 \leq \beta \leq 1$, whereas the variation of $\psi$ (z) is assumed to be $\psi(\mathrm{z})=\emptyset$, and at $\mathrm{z}=\mathrm{H}$ it is determined to be $\psi(\mathrm{z})=\varnothing_{0}$. So it can be expressed as

$$
\psi(z)=\varnothing_{0}+\left(\varnothing-\varnothing_{0}\right)\left(1-\frac{z}{H}\right) \beta
$$




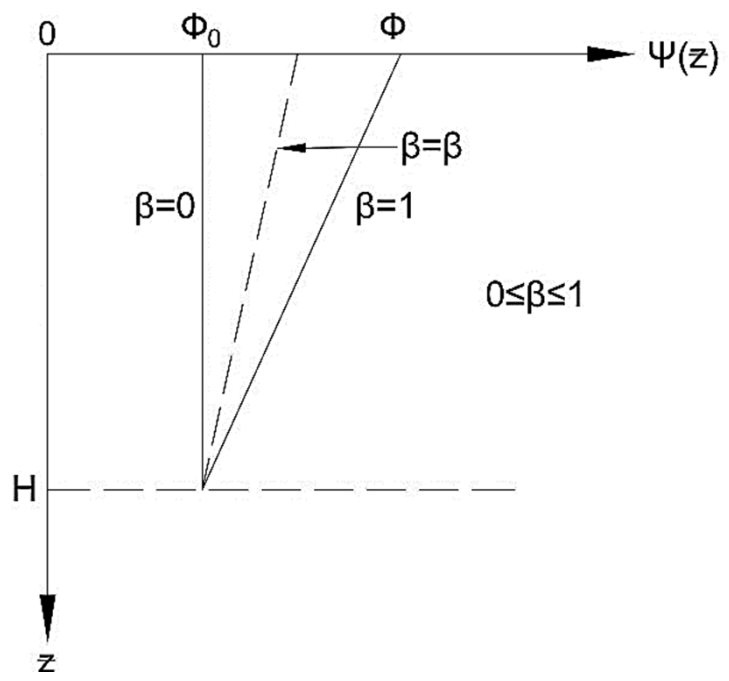

Figure 2: Transition of $\psi(z)$ from $\varnothing 0$ to $\varnothing$ with depth [10].

where

$$
\frac{\partial \psi(z)}{\partial z}=-\beta\left(\varnothing-\varnothing_{0}\right) / H
$$

Consequently, for $\beta=0$, at both depth $z=0$ and depth $\mathrm{z}=\mathrm{H}$, wall tilt is considered to be $\psi(\mathrm{z})=\varnothing_{0}$ and $\psi(\mathrm{z})=\varnothing$, respectively. The solution to $\beta=\beta$ at depth $\mathrm{z}=0$ can be expressed as $\emptyset_{0}<\psi(\mathrm{z})<\emptyset$, and at depth $\mathrm{z}=\mathrm{H} \psi(\mathrm{z})=\emptyset_{0}, \psi(\mathrm{z})$ decreases linearly between $\mathrm{z}=0$ and $\mathrm{z}=\mathrm{H}$. Using $\beta=1$ at depth $\mathrm{z}=0 \psi(\mathrm{z})=\varnothing$ and at depth $\mathrm{z}=\mathrm{H} \psi(\mathrm{z})=\emptyset_{0}$, the variation of $\psi(\mathrm{z})$ between $\mathrm{z}=0$ and $\mathrm{z}=\mathrm{H}$ reduces linearly.

\section{Application and Validation}

This section presents an assessment of the validity of the proposed model to a case of CSB slurry wall. A CSB is constructed to intercept and impede the stream of polluted fluids towards the groundwater. CSB provides lower structural strength compared to diaphragm and concrete walls. An alternative explanation might be that the CSB wall is principally constructed as a plastic medium with stressstrain properties much closer to the soil than concrete walls. Consequently, it should not be considered a structural wall, instead, the CSB slurry wall can be modeled as elastic soil [15]. On the basis of the relationship between stress and void ratio for CSB samples, Opdyke and
Evans [1] concluded that the coefficient of consolidation $\left(C_{v}\right)$ of $94 \times 10^{-4} \mathrm{~cm}^{2} / \mathrm{s}$ indicates the upper limit of natural clay soils, and the behavior is closely akin to overconsolidated soil. For remolded soils with liquid limits around $60 \%$, Sridharan and Nagaraj [16] have provided an upper limit for the coefficient of consolidation of $3 \times 10^{-4} \mathrm{~cm}^{2} / \mathrm{s}$. In addition, for marine clay treated with $8 \%$ lime and $20 \%$ slag, Rao and Raju [17] obtained a coefficient of consolidation of $5.17 \times 10^{-4} \mathrm{~cm}^{2} / \mathrm{s}$. In this study, the timerate of consolidation for CSB was investigated for loading increments from $200 \mathrm{kPa}$ to 400 $\mathrm{kPa}$. The laboratory experiment was conducted to investigate the consolidation parameters of the samples at 7 days and 28 days of curing time and other index properties (Table 1). Since the compressibility of CSB is similar to hard clay soil rather than a structural concrete wall, the authors decided to consider the performance of CSB as being similar to clay soil on the basis of elastic theory.

Laboratory datasets were assumed to apply on a site; that is, saturated medium dense sand encapsulated a trench with a width and a depth of $1.2 \mathrm{~m}$ and $30 \mathrm{~m}$, respectively. The Poisson's ratio values were theoretically obtained for the samples at a given curing time and are related to Young's modulus $E$ and elastic modulus $\mathrm{K}$ relationship, that is:

$$
\mathrm{E}=3 \mathrm{~K}(1-2 v)
$$

The Young's modulus $E$ of mixtures at each curing age has the following relationship with constrained modulus $\mathrm{D}$, corresponding to elastic theory

$$
E=\frac{(1+v)(1-2 v)}{1-v} D
$$

where

$$
D=\frac{\sigma^{\prime}}{\varepsilon}
$$

As the geotechnical status of the site shows, a direct relationship between the additional load module $k$ and the depth is used with the normal estimate of $n_{h}$ of submerged average thickness sand [13]. During the construction procedure, a 
Table 1. Consolidation and index properties.

\begin{tabular}{|c|c|c|c|c|c|}
\hline \multirow[t]{4}{*}{ Parameter } & \multirow[t]{4}{*}{ Unit } & \multicolumn{2}{|c|}{ C50-S50-B5 } & \multicolumn{2}{|c|}{ C50-S45-L5-B9 } \\
\hline & & \multicolumn{4}{|c|}{ Curing age (days) } \\
\hline & & 7 & 28 & 7 & 28 \\
\hline & & \multicolumn{4}{|c|}{ Value } \\
\hline Gs & / & \multicolumn{2}{|c|}{2.76} & \multicolumn{2}{|c|}{2.70} \\
\hline LL & $\%$ & \multicolumn{2}{|c|}{42} & \multicolumn{2}{|c|}{62} \\
\hline PI & $\%$ & \multicolumn{2}{|c|}{15} & \multicolumn{2}{|c|}{38} \\
\hline Clay Size & $\%$ & \multicolumn{2}{|c|}{17} & \multicolumn{2}{|c|}{17} \\
\hline Silt Size & $\%$ & \multicolumn{2}{|c|}{67} & \multicolumn{2}{|c|}{73} \\
\hline Sand Size & $\%$ & \multicolumn{2}{|c|}{16} & \multicolumn{2}{|c|}{10} \\
\hline$C_{v}$ & $\mathrm{~cm}^{2} / \mathrm{s}$ & $2.03 \times 10^{-3}$ & $1.24 \times 10^{-3}$ & $3.34 \times 10^{-4}$ & $3.0 \times 10^{-3}$ \\
\hline$\left(q_{u}\right)$ & $\mathrm{kPa}$ & 78 & 380 & 80 & 505 \\
\hline
\end{tabular}

backhoe or other specific equipment excavates the trench to the desired depth. At the same time, the excavated part of the trench is prevented from collapsing by pumping a bentonite-water slurry into it and keeping the trench full of batch slurry. Throughout the trench excavation, existing bentonite-water slurry penetrates through the sidewall into adjacent sandy layers. This bentonite filter cake then leads to reduced interface shear strength between the constructed wall and surrounding soils. Lam et al. [18] provided the strength of the shear reduction factor, $\mathrm{R}$, from calibrated CPTu data to be 0.1 to 0.3 .

Although the model provided by Li et al. [6] proposed a better estimation for $\sigma_{h^{\prime}}^{\prime}$ the $\sigma^{\prime}{ }_{v}$ achieved by this method was considered the major principal effective stress and was greater than $\sigma^{\prime}$. This approach is not well suited to deep trenches and may raise a question where the lateral squeezing effect is considered to be specified on the basis of Filz's [5] theory.

The development of lateral earth pressure behind a slurry wall creates inward displacement from mixture placement to consolidation completion. Laboratory investigation for increased strength of CSB also indicates that this deformation will stop to develop after 28 days of curing time because the unconfined compressive strength test values are greater than those provided by effective horizontal stress [19]. Vertical effective stress determination has various stages, starting from the at-rest condition (mixture placement) to the active condition (consolidation completion). In the analysis, the adjacent soil layer is assumed to have uniform properties with no change of layer type. The method of analysis estimates not only the progressive lateral earth pressure at each depth but also the dilatancy angle, $\psi$, development as $0 \leq \beta \leq 1$. The predicted vertical effective stress using this method is in good agreement with those provided by Ke et al. [8] and remains below effective horizontal stress at each depth. Moreover, by considering the effects of both arching and lateral squeezing, this model presents a considerable improvement of stress estimation in CSB slurry walls. The stress values obtained by the proposed model are used to estimate the permeability $(k)$ of the cured wall with depth through changes of the void ratio. The following affiliation between stress and permeability proposed by Yeo et al. [4] is used to exemplify the potential influence for samples of this study:

$$
e=1.25-0.21 \log \left(\frac{\sigma^{\prime}(k P a)}{5}\right)
$$

so that

$$
k(m / s)=1.5 \times 10^{-9} \times 10^{(e-1.25) / 0.22}
$$

where $\sigma^{\prime}$ and $e$ are effective consolidation stress and void ratio, respectively. Again, the conventional thinking is that the mechanical curing set provides one-dimensional (1D) stacking in CSB examples to control the proportion of gaps in 
each addition. In this way, the estimated effective stress obtained by the proposed model is adjusted by a comparable main effective pressure that would provide an inseparable fraction of the gap in the conditions of the 1D community, as indicated by Filz et al. [2]

$$
\sigma^{-1}=\frac{3 \sigma_{\text {mean }}^{\prime}}{1+2 K_{b}}
$$

where $\sigma^{-1}$ is the equivalent major effective stress, $\sigma_{\text {mean }}^{\prime}$ is the mean effective stress in cured samples, and $K_{\mathrm{b}}$ is assumed to be present as $\mathrm{u} /(1-\mathrm{v})$ for the consolidation test. Li et al. [6] indicated that the mean effective stress, $\sigma^{-1}$, can be adjusted for the plain-strain problem as follows:

$$
\sigma^{-1}=(1-v)\left(\sigma^{\prime}{ }_{v}+\sigma^{\prime}{ }_{h}\right)
$$

The estimated permeability profile controlled by the proposed effective stress at each depth is given in Figures 5 and 6 . In the shallow depth $(\mathrm{z} \leq 7 \mathrm{~m})$ where the arching effect is still dominant, permeability is higher than $10^{-9} \mathrm{~m} / \mathrm{s}$ as a result of lower effective stress and higher void ratio. The permeability decreases slightly with depth as stress increases and reaches $1 \times 10^{-9}$ $\mathrm{m} / \mathrm{s}$ in the region of $17 \mathrm{~m}$ and remains constant down to the trench's floor for the method proposed here. Predicted permeabilities for CSB in this study (Figures 5 and 6) appear to be well substantiated by those achieved in previous studies for both laboratory and field monitoring $[20,9]$. However, the permeability predicted by the geostatic method is lower than the presented method as a result of the greater stress values. According to the laboratory consolidation test results in this study, permeability decreases with depth as the void ratio decreases. Moreover, as specimens' hydration improve with increased curing times, the particles of the cementitious materials fill the void between them. Simultaneously, for the amended specimen, the presence of further bentonite content in addition to lime gave lower values than (C50-S50-B5) specimens for both curing times. The laboratory study also shows that permeability values for both mixtures at each curing period are slightly higher than those achieved by the proposed method (Figures 5 and 6).
The consolidometer permeation values in soft-to-medium consistency materials are 10 to 100 times greater than those gained by the flexible wall permeation test $[21,19]$.

\section{Parametric Study}

The proposed physical modeling in this study considers the effects of adjacent soil and mixture properties along with trench size on the steady-state effective stress profile. The values and the impact of corresponding parameters for both surrounded soils and mixtures are provided in Table 2. The width and depth of the trench are $1.2 \mathrm{~m}$ and $30 \mathrm{~m}$, respectively [8]. The laboratory investigation was performed to define the CSB mixture's properties and the impact of curing ages at 7 days and 28 days.

The values of subgrade modulus for granular soils $k$ correspond to saturated medium dense sand and varied with depth; the modulus of the horizontal subgrade reaction is defined as $n_{h}=4500 \mathrm{kN} / \mathrm{m}^{3}$ [13]. The impact of lateral squeezing throughout mixture consolidation and inward displacement is employed to determine $\sigma_{\mathrm{h}}^{\prime}$ in the CSB slurry wall according to depth.

The shear strength reduction factor at the interface of the mixture-adjacent soil also vary with depth and is $R=0.1-0.25$ according to data provided by Li et al. [6]. As $\beta$ indicates the wall stage at the elastic part of the inward displacement, the intermediate values for $\beta=1$ at $\mathrm{z}=0$ to $\beta=0$ at $\mathrm{z}=\mathrm{H}$ have been employed to determine $R$-value for each depth. Accordingly, the $C^{\prime}{ }_{\text {in }}$ and $\varnothing^{\prime}{ }_{\text {in }}$ parameters of the CSB wall are a function of $R$ and change with depth. The constrained modulus, $D$, for each mixture is determined on the basis of a one-dimensional consolidation test. Similarly, Young's modulus $E$ values for the samples are related to $D$ based on elasticity theory (see Equation 18). The impact of adjacent soils on effective vertical stress, $\sigma^{\prime}{ }_{v}$, is determined by using various values of $\emptyset_{C \mathrm{CSB}}{ }^{*}$ As discussed before, the minor effective stress, $\sigma_{\mathrm{v}^{\prime}}^{\prime}$ value is a function of $\beta, \varnothing^{\prime}{ }_{C S B}{ }^{\prime}$ and $\sigma^{\prime}{ }^{\prime}$, and must be determined for each depth. The described solution steps, however, need a parametric study to specify the impact of wall tilt, $\beta$, on the shear strength parameters. The values, as mentioned above for each parameter, are influenced by mixture properties at each curing age. 


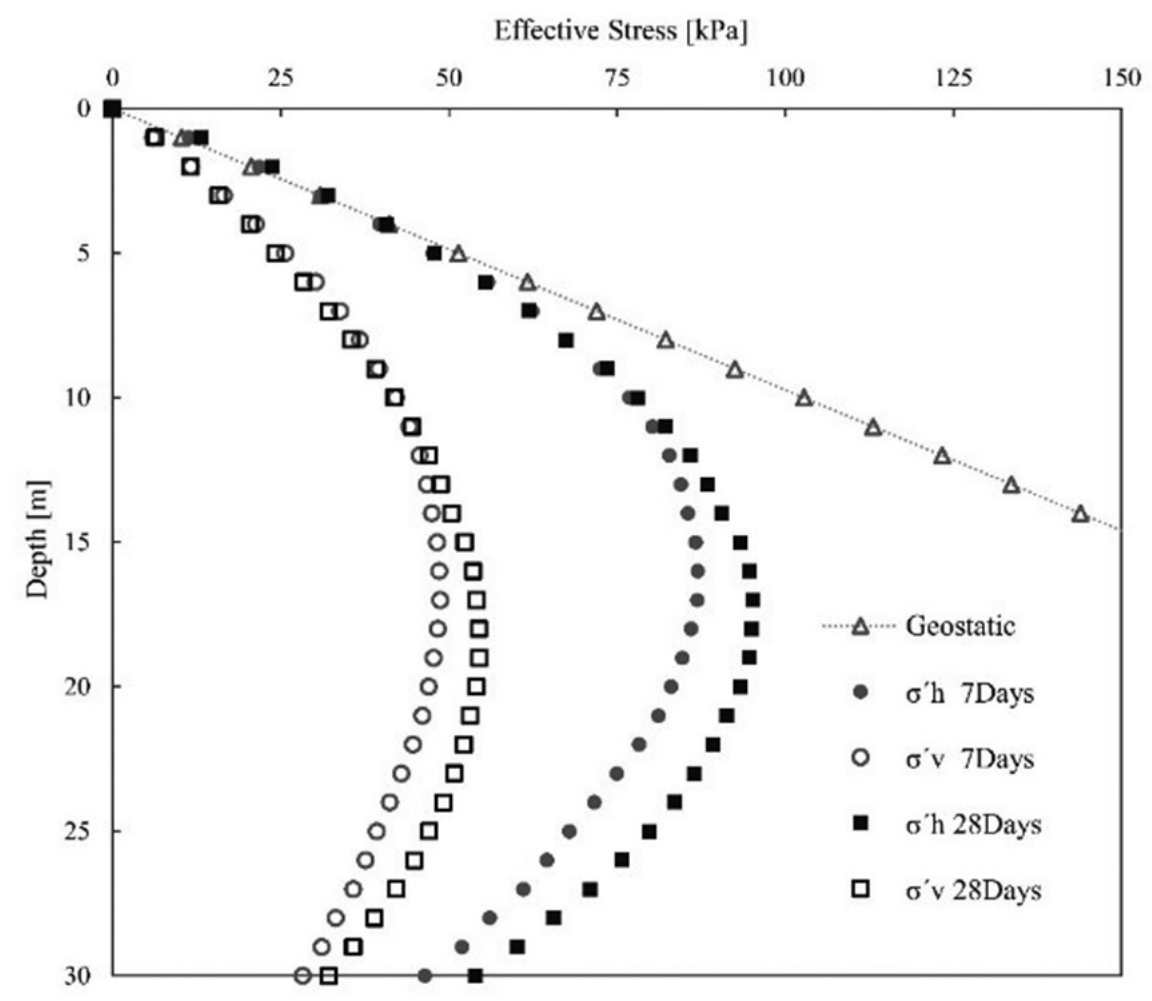

Figure 3: Effective stress values for the C50-S50-B5 mixture.

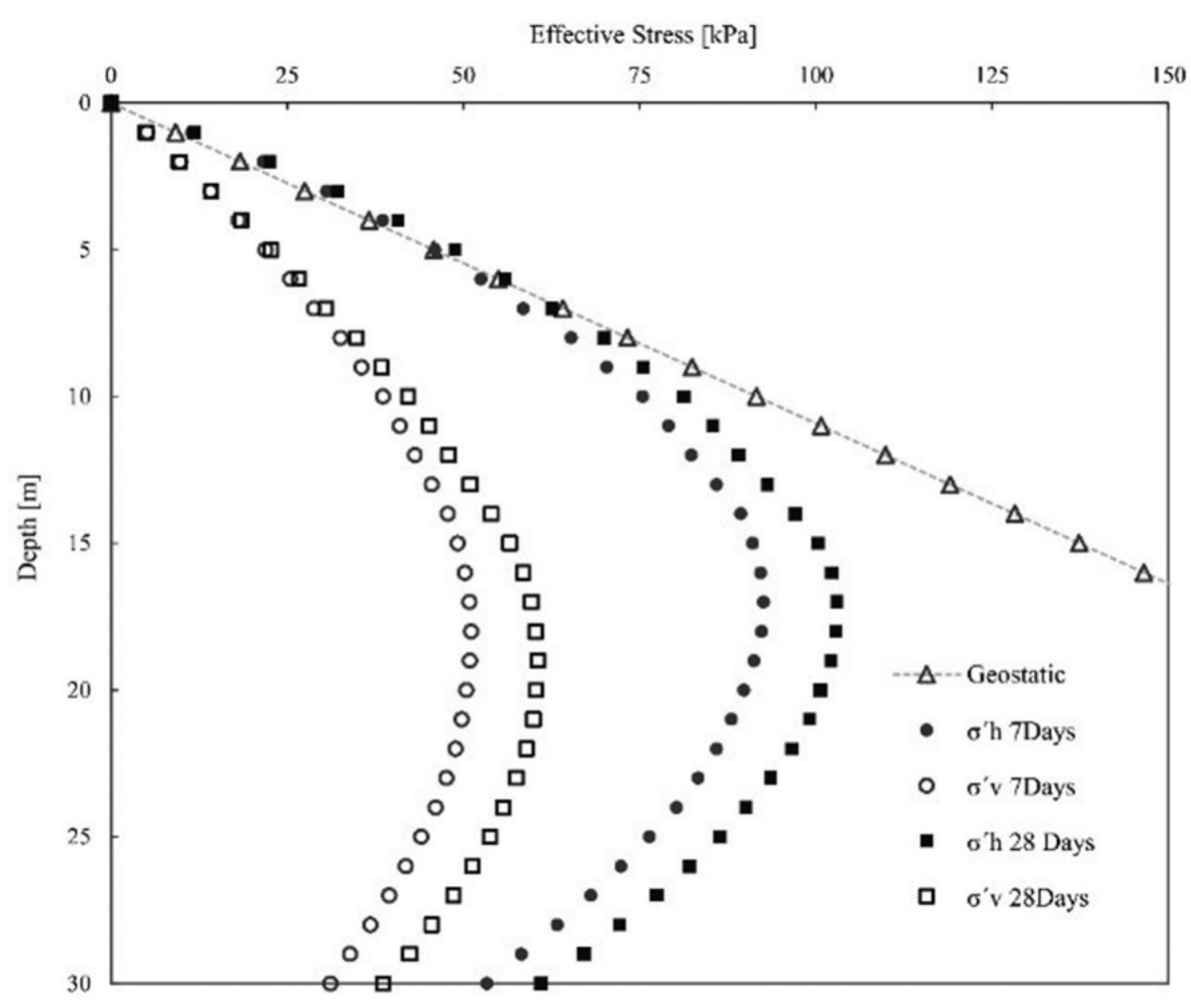

Figure 4: Effective stress values for the C50-S45-L5-B9 mixture. 


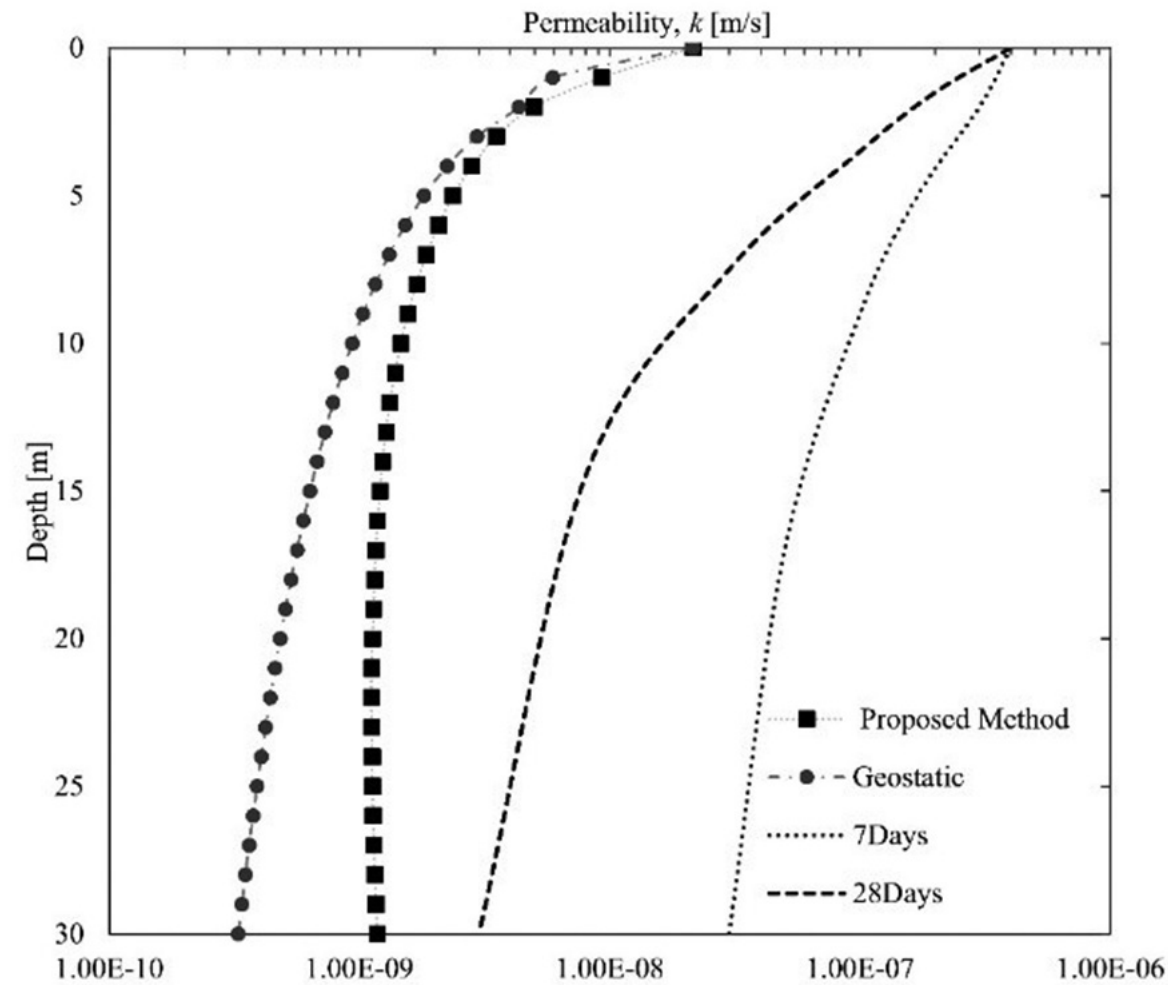

Figure 5: Estimated permeability and laboratory test results for the C50-S50-B5 mixture.

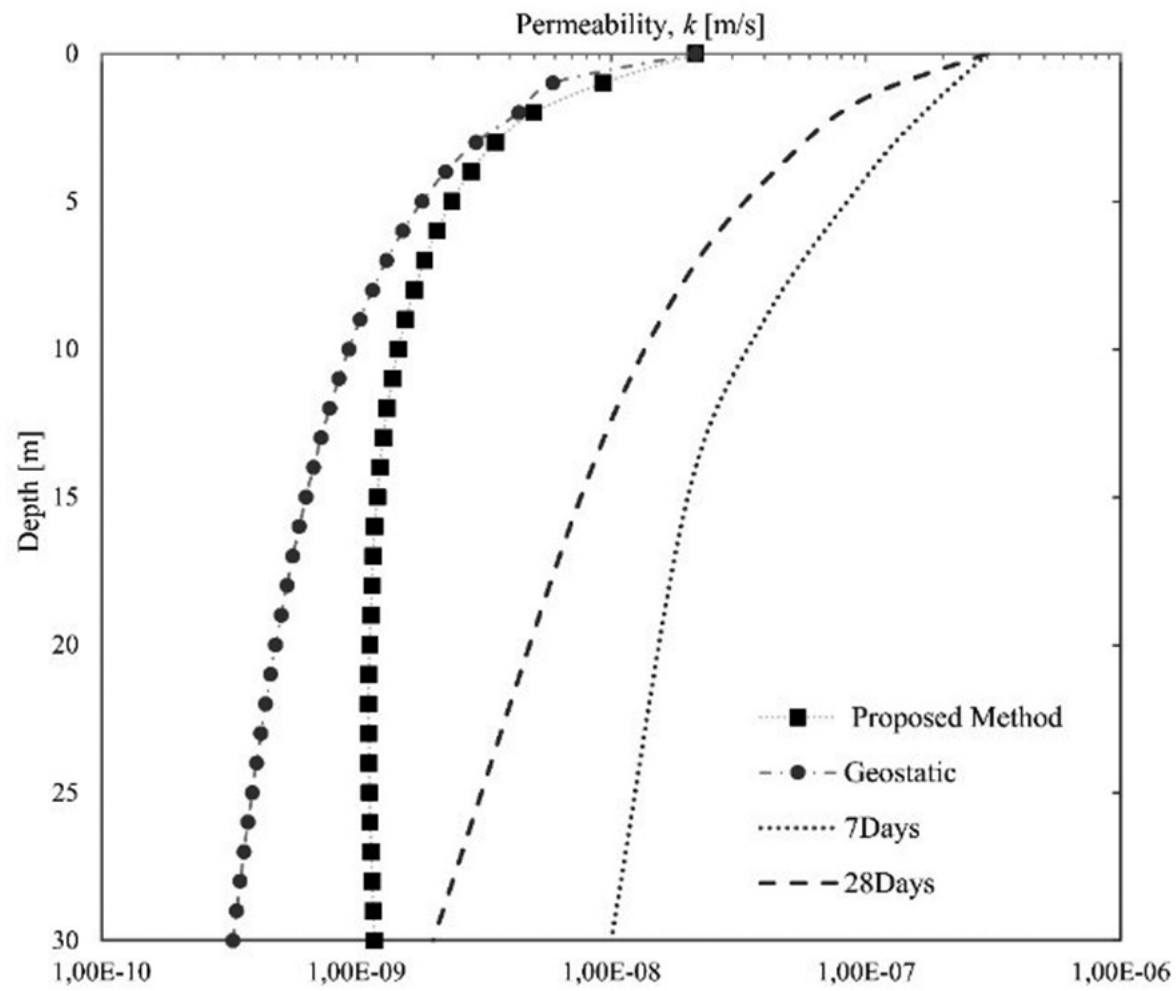

Figure 6: Estimated permeability and laboratory test results for the C50-S45-L5-B9 mixture. 
Table 2. Geometric and mixture properties.

\begin{tabular}{|c|c|c|c|c|c|}
\hline \multirow[t]{4}{*}{ Parameter } & \multirow[t]{4}{*}{ Unit } & \multicolumn{3}{|c|}{ C50-S50-B5 } & C50-S45-L5-B9 \\
\hline & & \multicolumn{4}{|c|}{ Curing age (days) } \\
\hline & & 7 & 28 & 7 & 28 \\
\hline & & \multicolumn{4}{|c|}{ Value } \\
\hline B & $\mathrm{m}$ & \multicolumn{4}{|c|}{$1.2^{*}$} \\
\hline $\mathrm{H}$ & $\mathrm{m}$ & \multicolumn{4}{|c|}{$30^{*}$} \\
\hline$\gamma_{\mathrm{csb}}^{\prime}$ & $\mathrm{kN} / \mathrm{m}^{3}$ & 11.9 & 13.8 & 12.3 & 12.6 \\
\hline v & / & 0.26 & 0.22 & 0.25 & 0.18 \\
\hline$C^{\prime}$ & $\mathrm{kPa}$ & 66 & 133 & 67 & 160 \\
\hline$\emptyset^{\prime}$ & $\circ$ & 18 & 21 & 23 & 25 \\
\hline $\mathrm{n}_{\mathrm{h}}$ & $\mathrm{kN} / \mathrm{m}^{3}$ & \multicolumn{4}{|c|}{$4500 \dagger$} \\
\hline$R$ & / & \multicolumn{4}{|c|}{$0.1-0.25 \ddagger$} \\
\hline
\end{tabular}

*Ke, $\mathrm{H}$ et al. 2018.

tDas 1998.

ఓLam et al. 2014.

\section{Discussion}

In this study, the effective horizontal stress, $\sigma^{\prime}{ }_{h^{\prime}}$ reduces owing to low lateral squeezing impact as $K$ increases linearly, and the maximum values are between $15 \mathrm{~m}$ to $20 \mathrm{~m}$ for both samples (Figures 3 and 4). The major and minor effective stress values for both samples are decreased as $\mathrm{z}>20 \mathrm{~m}$ and reached lower than $62 \mathrm{kPa}$ for the C50-S45-L5-B9 and $54 \mathrm{kPa}$ for C-50-S50-L5 mixtures at a depth of $30 \mathrm{~m}$. The parametric study also shows that the faster the hydration rate of a sample develops, the higher the effective stresses on the self-hardening walls. However, horizontal and vertical effective stress magnitude between two curing times do not exceed $10 \mathrm{kPa}$ for either mixture with depth. Besides, the properties of the CSB wall depend on mixture design and bentonite content. Considering reports from previous studies, this paper reveals that cementitious properties of slurry wall materials experience up to the $25 \mathrm{kPa}$ of major and minor effective stresses with depth. The laboratory tests revealed that the hydration progression of each sample has a slight influence on the proposed effective stress values to control the lateral squeezing effect. An alternative explanation might be that the increasing values of cohesion $C$ and internal friction angle $\emptyset$ of each mixture due to curing age does not have considerable impact on stress values. Measurements were taken as shear strength reduction factor $R$ changes with depth, that is, the function of $\beta$, and gives effective stress distribution values from the at-rest to the active condition. Moreover, the constrained modulus $D$ and Young's modulus $E$ values of the CSB mixtures are stress dependent and increase with depth. The results show that since the differences between the maximum and minimum value of $E$ for both mixtures at 7-day and 28-day curing ages are relatively low, it does not have a major impact on the effective stress in the wall. The coefficient of lateral earth pressure has a significant effect on the vertical effective stress prediction. Ke et al. [8] indicated that this prediction accuracy could be improved by inputting a distribution of the lateral earth pressure coefficient with depth.

Such an investigation is developed in Equation 15, and the results are based on the determination of the intermediate value of $\varnothing$ and $\emptyset_{0}$ at each depth. The proposed physical model provides a relatively good estimation of the stress distribution within CSB slurry walls. The trends of both major and minor effective stress achieved by this model are in good agreement with those calculated from CPTu data sets provided by Ke et al. [8]. The proposed stresses are used to estimate the permeability of the CSB wall and compare it with those evaluated in the laboratory for the same stress profile. Estimated permeability and laboratory test 
results for the C50-S50-B5 mixture and the C50S45-L5-B9 mixture are presented in Figures 5 and 6 . The permeability results of both would seem to suggest that permeability is relatively high in the shallow depth where stress is lower and it decreases as stress increases.

The use of vertical curtains in order to contain the advance of contaminating plumes towards groundwater is increasing throughout the world [19]. These are trench-shaped excavations that can reach up to tens of meters in depth and are strategically placed to contain, diverge, or encapsulate the underground contaminating flow. Simultaneously with the excavation process, the trench is filled with a mixture of bentonite and water, called bentonite mud, which has functions of geotechnical stabilization and reduced permeability [7]. After the excavation, the trench is re-ground, so that part of the introduced bentonite mud is expelled, and the remaining part tends to remain, covering the walls and the bottom of the ditch and forming a thin layer (filter cake) [2].

This thin layer, if well constituted, is mainly responsible for the reduction of the permeability of the curtain [11]. The backfill, normally constituted of the excavated material itself, can be mixed with bentonite and/or cement, in order to further reduce its permeability and increase its mechanical resistance. Geomembranes can also be applied next to the backfill to further decrease the hydraulic conductivity of the curtain [2]. Lateritic soils, in turn, cover approximately $65 \%$ of the country's territory. In general, they are characterized by a mineralogical composition dominated by quartz, Fe-Al-Mn oxides/hydroxides, and kaolinite as the predominant clay. In the field, they usually present high porosity and drainability, in profiles of alteration of great thicknesses, and are quite homogeneous. Because of its wide occurrence, lateritic soils have great potential for application in vertical curtains.

\section{Conclusion}

This paper has investigated the physical model to predict effective stresses in CSB slurry walls, and considerable progress has been made for both arching and lateral squeezing effect. Taken together, the proposed model was then employed to evaluate the influence of two different self-hardening mixtures on estimated stress profile. A bentonite filter cake prior to mixture hardening reduces the shear strength between adjacent soils and the trench wall, and the provided $R, \mathrm{C}_{\mathrm{CSB}^{\prime}}$, and $\emptyset_{\mathrm{CSB}}^{\prime}$ values change with depth according to $\beta$ tilt. The principal stress of the trench wall at $\mathrm{z}>$ $7 \mathrm{~m}$ is horizontal, and maximum stress values are appear at $15 \leq \mathrm{z} \leq 20 \mathrm{~m}$. A parametric study implies that the stresses in the CSB wall are initially dominated by the arching effect at shallow depth; later at $\mathrm{z} \geq 7 \mathrm{~m}$, stresses are caused by lateral squeezing. The effective vertical stresses are then predicted based on obtained horizontal effective stresses and the transition of the lateral earth pressure coefficient from at-rest to the active condition. The proposed stresses are used to estimate the permeability of the CSB wall and compare it with those evaluated in the laboratory for the same stress profile. The permeability results of both would seem to suggest that permeability is relatively high for the shallow depth as a result of its low-stress stage, and permeability decreases as stress increases. Finally, considerable insight has been gained with regard to an appropriate stress stage prediction in the CSB wall, in which the development is consistent with calculations from CPTu data provided by previous studies.

\section{References}

[1] Opdyke, S.M., Evans, J.C. (2005): Slag-cementbentonite slurry walls. Journal of Geotechnical and Geoenvironmental Engineering, 131(6), pp. 673-681.

[2] Filz, G.M., Henry, L.B., Heslin, G.M., Davidson, R.R. (2001): Determining hydraulic conductivity of soil-bentonite using the API filter press. Geotechnical Testing Journal, 24(1), pp. 61-71.

[3] Evans, J. C. (1994): Hydraulic conductivity of vertical cutoff walls. In: Hydraulic Conductivity and Waste Contaminant Transport in Soil. Daniel, D, E, Trautwein, S.J. (eds.), ASTM International: West Conshohocken, Pennsylvania.

[4] Yeo, S.S., Shackelford, C.D., Evans, J.C. (2005): Consolidation and hydraulic conductivity of nine model soil-bentonite backfills. Journal of 
Geotechnical and Geoenvironmental Engineering, 131(10), pp. 1189-1198.

[5] Filz, G.M., 1996. Consolidation stresses in soil-bentonite backfilled trenches. In: Environmental Geotechnics, Kamon, M. (ed.). CRC Press: Boca Raton, Florida, pp. 497-502.

[6] Li, Y.C., Cleall, P.J., Wen, Y.D., Chen, Y.M., Pan, Q. (2015): Stresses in soil-bentonite slurry trench cutoff walls. Géotechnique, 65(10), pp. 843-850.

[7] Ruffing, D.G., Evans, J.C., Malusis, M.A. (2010): Prediction of earth pressures in soil-bentonite cutoff walls. In: GeoFlorida 2010: Advances in Analysis, Modeling \& Design, Fratta, D.O., Pupala, A.J., Muhunthan, B. (eds), ASCE Library: Reston, Virginia, pp. 2416-2425.

[8] Ke, H., Tong, X., Li, Y.C., Chen, Y.M., Wen, Y.D. (2018): Force equilibrium-based model for predicting stresses in soil-bentonite cutoff walls. Journal of Geotechnical and Geoenvironmental Engineering, 144(2), p.04017112.

[9] Ruffing, D. G., Evans, J.C. (2014): Case study: construction and in situ hydraulic conductivity evaluation of a deep soil-cement-bentonite cutoff wall. In: Geo-Congress 2014: Geo-Characterization and Modeling for Sustainability, Abu-Farsakh, M., Yu, X., Hoyos, L.R. (eds.), ASCE Library: Reston, Virginia, pp. 1836-1848.

[10] Bang, S., Kim, H.T. (1986): At rest to active earth pressure transition. Transportation Research Record, 1105, pp. 41-47.

[11] Evans, J.C., Fang, H.Y., Kugelman, I.J. (1985): Containment of hazardous materials with soil-bentonite slurry walls. In: Proceedings of the 6th National Conference on the Management of Uncontrolled Hazardous Waste Sites, United States Environmental Protection Agency: Silver Spring, Maryland, pp. 249-252.
[12] Potyondy, J.G. (1961): Skin friction between various soils and construction materials. Geotechnique, 11(4), pp. 339-353.

[13] Das, B.M. (1998): Principles of Foundation Engineering. Brooks/Cole: Pacific Grove, California.

[14] Clough, G.W., Duncan, J.M. (1991): Earth pressures. In: Foundation Engineering Handbook, Fang H.Y. (ed.). Springer Science-Business Media: New York.

[15] Nejad, B.G., Osborne, T., Carter, J.P. (2017): Forensic Investigation of a Slurry Wall Failure: A Case Study. In: Grouting, ASCE Library: Reston, VA, USA, pp. 513-522.

[16] Sridharan, A., Nagaraj, H.B. (2004): Coefficient of consolidation and its correlation with index properties of remolded soils. Geotechnical Testing Journal, 27, 469-474.

[17] Rao, D.K., Raju, R.P. \& Kumar, R.A. (2011): Consolidation characteristics of treated marine clay for foundation bed soils. International Journal of Engineering Science and Technology. 3(2), pp. 788-796.

[18] Lam, C., Jefferis, S.A., Martin, C.M. (2014): Effects of polymer and bentonite support fluids on concretesand interface shear strength. Géotechnique, 64(1), pp. 28-39.

[19] Talefirouz, D., Cokça, E., Omer, J. (2016): Use of granulated blast furnace slag and lime in cement-bentonite slurry wall construction. International Journal of Geotechnical Engineering, 10(1), pp. 81-85.

[20] Boscardin, M., Patterson, C., Landis, M., Younan, J. C., Aghjayan, D, (2006): Evaluation of permeability of containment slurry walls. In: GeoCongress 2006: Geotechnical Engineering in the Information Technology Age, DeGroot, D., DeJong, J., Frost, D., Baise, L. (eds.). American Society of Civil Engineers: Reston, Virginia, pp. 1-6.

[21] Oweis, I.S., Khera, R.P. (1990): Geotechnology of Waste Management. Butterworth-Heineman: Oxford, UK. 